\title{
A BORSUK-ULAM TYPE THEOREM OVER ITERATED SUSPENSIONS OF REAL PROJECTIVE SPACES
}

\author{
RYUICHI TANAKA
}

\begin{abstract}
A CW complex $B$ is said to be I-trivial if there does not exist a $\mathbb{Z}_{2}$-map from $S^{i-1}$ to $S(\alpha)$ for any vector bundle $\alpha$ over $B$ and any integer $i$ with $i>\operatorname{dim} \alpha$. In this paper, we consider the question of determining whether $\Sigma^{k} \mathbb{R} P^{n}$ is I-trivial or not, and to this question we give complete answers when $k \neq 1,3,8$ and partial answers when $k=1,3,8$. A CW complex $B$ is I-trivial if it is "W-trivial", that is, if for every vector bundle over $B$, all the Stiefel-Whitney classes vanish. We find, as a result, that $\Sigma^{k} \mathbb{R} P^{n}$ is a counterexample to the converse of this statement when $k=2,4$ or 8 and $n \geq 2 k$.
\end{abstract}

\section{Introduction and results}

For a real vector bundle $\alpha$ over a $\mathrm{CW}$ complex $B$, the index of $\alpha$, denoted by ind $\alpha$, is defined to be the largest integer $i$ for which there exists a $\mathbb{Z}_{2}$-map from the $(i-1)$-sphere $S^{i-1}$ to $S(\alpha)$ (see $[1,2,6]$ ). Here, $S(\alpha)$ is the sphere bundle of $\alpha$ and it is regarded as $\mathbb{Z}_{2}$-space by the antipodal map on each fiber. The sphere $S^{i-1}$ is also regarded as $\mathbb{Z}_{2}$-space by the antipodal map. Obviously we have the inequality ind $\alpha \geq \operatorname{dim} \alpha$ for any $\alpha$. The underlying space $B$ is said to be I-trivial if the equality ind $\alpha=\operatorname{dim} \alpha$ holds for every vector bundle $\alpha$ over $B$. With this terminology, the classical Borsuk-Ulam theorem can be restated as the point space is I-trivial. Also, the sphere $S^{n}$ is I-trivial if and only if $n \neq 1,2,4,8$ (see $[5,7]$ ). As for the stunted projective space $F P_{m}^{n}$, where $F=\mathbb{R}, \mathbb{C}$ or $\mathbb{H}$, with a function $\lambda_{F}(n)$ suitably defined, it is shown in $[9]$ that $F P_{m}^{n}$ is I-trivial if and only if $\lambda_{F}(n)<m \leq n$. Note that in [9] the symbol $\theta(n)$ is used instead of $\lambda_{\mathbb{R}}(n)$.

In this paper, we investigate whether, for positive integers $k$ and $n$, the $k$-fold suspension $\Sigma^{k} \mathbb{R} P^{n}$ of $\mathbb{R} P^{n}$ is I-trivial or not.

In general, for a vector bundle $\alpha$, we have ind $\alpha=\operatorname{dim} \alpha$ if the total StiefelWhitney class $W(\alpha)$ is equal to 1 (see [6, Proposition 2.2]). The underlying space $B$ is said to be $\mathrm{W}$-trivial if $W(\alpha)=1$ holds for every vector bundle $\alpha$ over

Received October 3, 2010.

2010 Mathematics Subject Classification. Primary 55P91; Secondary 55R25.

Key words and phrases. sphere bundle, $\mathbb{Z}_{2}$-map, index. 
$B$. Thus, we immediately have the following lemma, which is of fundamental importance in our study.

Lemma 1.1. If $B$ is $W$-trivial, then it is I-trivial.

Concerning whether $\Sigma^{k} \mathbb{R} P^{n}$ is W-trivial or not, we quote the following result from $[10]$.

Theorem 1.2 ([10, Theorem 1.4]). For positive integers $k$ and $n$, the $k$-fold suspension $\Sigma^{k} \mathbb{R} P^{n}$ of $\mathbb{R} P^{n}$ is not $W$-trivial if and only if $k$ and $n$ satisfy one of the following conditions:

(1) $k=1,2,4$ or 8 and $n \geq k$.

(2) $k=3,5$ or 7 and $n+k=4$ or 8 .

(3) $k=6$ and $n=2$ or 3 .

Accordingly, our goal is to determine whether or not $\Sigma^{k} \mathbb{R} P^{n}$ is I-trivial for each pair $(k, n)$ as in the above theorem. The results obtained in this paper are summarized as the theorem below. Note that if $k \geq 9, \Sigma^{k} \mathbb{R} P^{n}$ is I-trivial for all $n \geq 1$ since it is $\mathrm{W}$-trivial.

Theorem 1.3. Let $n$ be a positive integer.

(1) $\Sigma \mathbb{R} P^{n}$ is not I-trivial if $n=1,2$ or 3 .

(2) $\Sigma^{2} \mathbb{R} P^{n}$ is not I-trivial if and only if $n=2$ or 3 .

(3) $\Sigma^{3} \mathbb{R} P^{n}$ is not I-trivial only if $n=1$ or 5 . It is not I-trivial if $n=1$.

(4) $\Sigma^{4} \mathbb{R} P^{n}$ is not I-trivial if and only if $4 \leq n \leq 7$.

(5) $\Sigma^{5} \mathbb{R} P^{n}$ is not I-trivial if and only if $n=3$.

(6) $\Sigma^{6} \mathbb{R} P^{n}$ is not I-trivial if and only if $n=2$ or 3 .

(7) $\Sigma^{7} \mathbb{R} P^{n}$ is not I-trivial if and only if $n=1$.

(8) $\Sigma^{8} \mathbb{R} P^{n}$ is not I-trivial only if $8 \leq n \leq 15$. It is not I-trivial if $n=8$.

Thus, the following three cases remain still unsettled: (i) $k=1$ with $n \geq 4$, (ii) $k=3$ with $n=5$, and (iii) $k=8$ with $8<n \leq 15$.

The next corollary, which is an immediate consequence of Theorems 1.2 and 1.3, shows that the converse of Lemma 1.1 is not always true.

Corollary 1.4. The following spaces are I-trivial, although they are not $W$ trivial.

(1) $\Sigma^{2} \mathbb{R} P^{n}$ with $n \geq 4$

(2) $\Sigma^{4} \mathbb{R} P^{n}$ with $n \geq 8$.

(3) $\Sigma^{8} \mathbb{R} P^{n}$ with $n \geq 16$.

Throughout this paper, all cohomology is assumed to have coefficients $\mathbb{Z}_{2}$ unless otherwise stated. We often consider a homomorphism from $H^{*}\left(\Sigma^{k} \mathbb{R} P^{n}\right)$ to $H^{*}\left(\mathbb{R} P^{m}\right)$. We denote by $s^{k} a^{i}$ the non-zero element of $H^{k+i}\left(\Sigma^{k} \mathbb{R} P^{n}\right)(1 \leq$ $i \leq n)$, where $a$ represents the generator of $H^{*}\left(\mathbb{R} P^{n}\right)$ and $s^{k}$ represents $k$-fold suspension. Besides this notation, we use the letter $t$ to denote the generator of 
$H^{*}\left(\mathbb{R} P^{m}\right)$ for $m$ not necessarily equal to $n$. $\operatorname{Hom}\left(\widetilde{H}^{*}\left(\Sigma^{k} \mathbb{R} P^{n}\right), \widetilde{H}^{*}\left(\mathbb{R} P^{m}\right)\right)$ denotes the group consisting of all homomorphisms (of degree 0 ) from $\widetilde{H}^{*}\left(\Sigma^{k} \mathbb{R} P^{n}\right)$ to $\widetilde{H}^{*}\left(\mathbb{R} P^{m}\right)$ as graded algebra over the Steenrod algebra mod 2.

Also, it is to be understood that the notation $\xi$ always denotes the canonical line bundle over $\mathbb{R} P^{n}$ (for various values of $n$ ).

\section{Preliminaries}

Let $B$ be a CW complex. The following proposition, which will be used to show that $B$ is I-trivial, is a slight generalization of Proposition 2.4 of [6].

Proposition 2.1. Let $\alpha$ be a vector bundle over $B$ with $\operatorname{dim} \alpha=m$ and let $k$ be an integer with $m \leq k$.

(1) If $g^{*}(W(\alpha))=1$ for any map $g: \mathbb{R} P^{k} \longrightarrow B$, then we have $m \leq$ ind $\alpha \leq k$.

(2) If $g^{*}(W(\alpha))=1$ for any map $g: \mathbb{R} P^{m} \longrightarrow B$, then we have ind $\alpha=m$.

Proof. Part (2) is the special case when $k=m$ in part (1). We prove part (1). Assume that ind $\alpha>k$. Then there exists a $\mathbb{Z}_{2}$-map $f: S^{k} \rightarrow S(\alpha)$. We consider the induced map $\tilde{f}: \mathbb{R} P^{k} \rightarrow P(\alpha)$, where $P(\alpha)$ denotes the associated projective bundle of $\alpha$. Denoting by $e$ the $\mathbb{Z}_{2}$-Euler class of the line bundle $\alpha \rightarrow P(\alpha)$, we have $\tilde{f}^{*}(e)=t$, where $t$ is the generator of $H^{*}\left(\mathbb{R} P^{k}\right)$. In $H^{m}(P(\alpha))$, there is a well-known relation as follows:

$$
e^{m}=\sum_{i=0}^{m-1} w_{m-i}(\alpha) e^{i}
$$

Applying $\tilde{f}^{*}$ to this relation, we obtain the following relation in $H^{m}\left(\mathbb{R} P^{k}\right)$ :

$$
t^{m}=\sum_{i=0}^{m-1} g^{*}\left(w_{m-i}(\alpha)\right) t^{i},
$$

where $g$ denotes the composite of $\tilde{f}$ and the projection $p: P(\alpha) \rightarrow B$. Here, if we suppose that $g^{*}(W(\alpha))=1$, that is, $g^{*}\left(w_{i}(\alpha)\right)=0$ for all $i>0$, then the above relation is reduced to $t^{m}=0$. However, we have $t^{m} \neq 0$ since $m \leq k$. This is a contradiction, so that the proof of Proposition 2.1 is completed.

Remark. In the above proposition, the assumption of (1) holds especially when $\operatorname{Hom}\left(\widetilde{H}^{*}(B), \widetilde{H}^{*}\left(\mathbb{R} P^{k}\right)\right)=0$. This gives Proposition 2.4 of $[6]$. Also, the assumption of (2) trivially holds when $W(\alpha)=1$. This gives Proposition 2.2 of [6].

The following proposition will be used to show that $B$ is not I-trivial.

Proposition 2.2. Let $d=2,4$ or 8 , and let $\rho_{d}$ denote the Hopf vector bundle over $S^{d}$. Assume there exist maps $f: B \longrightarrow S^{d}$ and $g: \mathbb{R} P^{d} \longrightarrow B$ such that

$$
(f \circ g)^{*}: H^{d}\left(S^{d}\right) \longrightarrow H^{d}\left(\mathbb{R} P^{d}\right)
$$


is an isomorphism. Then we have ind $f^{*}\left(\rho_{d}\right)>d$. Consequently, $B$ is not I-trivial.

Proof. Let us consider the sequence of maps

$$
\mathbb{R} P^{d \stackrel{g}{\longrightarrow}} B \stackrel{f}{\longrightarrow} S^{d}
$$

and the Hopf vector bundle $\rho_{d}$ over $S^{d}$. We put $\alpha=f^{*}\left(\rho_{d}\right)$ and prove that ind $\alpha>\operatorname{dim} \alpha(=d)$. Let $s$ and $t$ be the generators of $H^{d}\left(S^{d}\right)$ and $H^{1}\left(\mathbb{R} P^{d}\right)$ respectively. We assume that $(f \circ g)^{*}$ is an isomorphism. Then, we have

$$
\begin{aligned}
W\left(g^{*}(\alpha)\right) & =(f \circ g)^{*} W\left(\rho_{d}\right) \\
& =(f \circ g)^{*}(1+s) \\
& =1+t^{d} \\
& =(1+t)^{d},
\end{aligned}
$$

since $d$ is a power of 2 . Let $\xi$ be the canonical line bundle over $\mathbb{R} P^{d}$. Since $W(\xi)=1+t$, we have $W\left(g^{*}(\alpha) \otimes \xi\right)=(1+t+t)^{d}=1$ by an analogous formula to Formula III of Theorem 4.4.3 in [4]. Thus, we see that $g^{*}(\alpha) \otimes$ $\xi$ is orientable and the only obstruction to its non-zero cross section lies in $H^{d}\left(\mathbb{R} P^{d} ; \pi_{d-1}\left(S^{d-1}\right)\right)$. This obstruction vanishes, since $w_{d}\left(g^{*}(\alpha) \otimes \xi\right)=0$ and since the $\bmod 2$ reduction $H^{d}\left(\mathbb{R} P^{d} ; \mathbb{Z}\right) \rightarrow H^{d}\left(\mathbb{R} P^{d}\right)$ is an isomorphism. Therefore, we can decompose $g^{*}(\alpha) \otimes \xi$ into the form $1 \oplus \beta$ for some vector bundle $\beta$ with $\operatorname{dim} \beta=d-1$. Tensoring with $\xi$, we have $g^{*}(\alpha)=\xi \oplus(\beta \otimes \xi)$, so that we obtain the bundle monomorphism

$$
\xi \hookrightarrow g^{*}(\alpha) \stackrel{g}{\longrightarrow} \alpha .
$$

Restricting this bundle monomorphism to the sphere bundles, we obtain a $\mathbb{Z}_{2^{-}}$ $\operatorname{map} S(\xi) \rightarrow S(\alpha)$. Since $S(\xi)=S^{d}$, we have ind $\alpha \geq d+1$ and the proposition follows.

\section{3. $k$-fold suspension for the case $k=2,4$ or 8}

In this section, we consider $\Sigma^{k} \mathbb{R} P^{n}$ with $k=2,4$ or 8 and prove parts (2), (4) and (8) of Theorem 1.3. We write $d$ instead of $k$. If $n<d$, then $\Sigma^{d} \mathbb{R} P^{n}$ is $\mathrm{W}$-trivial by Theorem 1.2, so it is I-trivial. Therefore, we consider only the case $n \geq d$ hereafter. We remark that especially in the case where $n=d=8$, $\Sigma^{d} \mathbb{R} P^{n}$ is seen to be not I-trivial. In fact, denoting by $\xi$ the canonical line bundle over $\mathbb{R} P^{7}$, we have

$$
\Sigma^{8} \mathbb{R} P^{8}=\Sigma^{8}\left(\mathbb{R} P^{7}\right)^{\xi}=\left(\mathbb{R} P^{7}\right)^{\xi+8}=\left(\mathbb{R} P^{7}\right)^{9 \xi}=\mathbb{R} P_{9}^{16},
$$

which is not I-trivial by Theorem 1.2 of [9]. Thus the second half of (8) follows. We prove the following theorem, which immediately leads to parts (2), (4) and the first half of (8).

Theorem 3.1. Let $d=2,4$ or 8 .

(1) If $n \geq 2 d$, then $\Sigma^{d} \mathbb{R} P^{n}$ is I-trivial. 
(2) If $d \leq n \leq 2 d-1$, then we have the following:

(a) For any vector bundle $\alpha$ over $\Sigma^{d} \mathbb{R} P^{n}$ with $\operatorname{dim} \alpha \neq 2 d$, we have ind $\alpha=\operatorname{dim} \alpha$.

(b) For a vector bundle $\alpha$ over $\Sigma^{d} \mathbb{R} P^{n}$ with $\operatorname{dim} \alpha=2 d$, we have ind $\alpha=2 d$ or $2 d+1$.

(c) In the case where $d=2$ or 4 , there exists a vector bundle $\alpha$ over $\Sigma^{d} \mathbb{R} P^{n}$ with $\operatorname{dim} \alpha=2 d$ such that ind $\alpha=2 d+1$; consequently, $\Sigma^{d} \mathbb{R} P^{n}$ is not I-trivial.

The above theorem can be equivalently rewritten as the following two propositions.

Proposition 3.2. Let $d=2,4$ or 8 (and $n \geq d$ ). Let $\alpha$ be a vector bundle over $\Sigma^{d} \mathbb{R} P^{n}$ with $\operatorname{dim} \alpha=m$.

(1) If $m \neq 2 d$, then we have ind $\alpha=m$.

(2) If $m=2 d$, then we have ind $\alpha=m$ or $m+1$, and in particular, we have ind $\alpha=m$ in the case $n \geq 2 d$.

Proposition 3.3. Let $d=2$ or 4 , and assume that $d \leq n \leq 2 d-1$. Then there exists a vector bundle $\alpha$ over $\Sigma^{d} \mathbb{R} P^{n}$ with $\operatorname{dim} \alpha=2 d$ such that ind $\alpha=2 d+1$.

To prove Proposition 3.2, we prepare the following lemma, in which we merely suppose that $d$ is even and we do not necessarily require that $d=2,4$ or 8 .

Lemma 3.4. Let $d, n$, and $m$ be positive integers with $d$ even. Assume that $m \geq 2 d+1$.

(1) If $m-d+1$ is not a power of 2 , we have

$$
\operatorname{Hom}\left(\widetilde{H}^{*}\left(\Sigma^{d} \mathbb{R} P^{n}\right), \widetilde{H}^{*}\left(\mathbb{R} P^{m}\right)\right)=0 .
$$

(2) If $m-d+1$ is a power of 2 , we have

$$
\operatorname{Hom}\left(\widetilde{H}^{*}\left(\Sigma^{d} \mathbb{R} P^{n}\right), \widetilde{H}^{*}\left(\mathbb{R} P^{m}\right)\right) \cong \begin{cases}0 & (m>n+d), \\ \mathbb{Z}_{2} & (m \leq n+d),\end{cases}
$$

and in the latter case, the non-zero homomorphism is non-zero only in dimension $m$.

Proof. Let $\varphi: \widetilde{H}^{*}\left(\Sigma^{d} \mathbb{R} P^{n}\right) \rightarrow \widetilde{H}^{*}\left(\mathbb{R} P^{m}\right)$ be a homomorphism. For a nonnegative integer $r$, we claim that the following three statements are true:

(i) $\varphi\left(s^{d} a^{2^{r}}\right)=0$.

(ii) If $2^{r}-1+d \neq m$ and $r \geq 2$, then $\varphi\left(s^{d} a^{2^{r}-1}\right)=0$.

(iii) If $\varphi\left(s^{d} a^{2^{r}-1}\right)=0$ and $r \geq 2$, then $\varphi\left(s^{d} a^{i}\right)=0$ for $2^{r}+1 \leq i \leq 2^{r+1}-2$.

We first show statement (i). To show $\varphi\left(s^{d} a^{2^{r}}\right)=0$ for $r=0$, we use the Steenrod square $S q^{d}$. We clearly have $S q^{d}\left(s^{d} a\right)=s^{d} S q^{d} a=0$. On the other hand, we have $S q^{d}\left(t^{d+1}\right)=\left(\begin{array}{c}d+1 \\ d\end{array}\right) t^{2 d+1} \neq 0$ since $d$ is even and $2 d+1 \leq m$. 
Therefore we obtain $\varphi\left(s^{d} a\right) \neq t^{d+1}$, that is, $\varphi\left(s^{d} a\right)=0$. For $r \geq 1$, we also have $\varphi\left(s^{d} a^{2^{r}}\right)=0$ since $a^{2^{r}}=S q^{2^{r-1}} S q^{2^{r-2}} \cdots S q^{1} a$.

Next we show statement (ii). If $2^{r}-1+d>m$, we clearly have $\varphi\left(s^{d} a^{2^{r}-1}\right)=$ 0 in $\widetilde{H}^{*}\left(\mathbb{R} P^{m}\right)$ for dimensional reasons. So let $2^{r}-1+d<m$. We use $S q^{1}$ and the result of (i). First, we have $S q^{1}\left(s^{d} a^{2^{r}-1}\right)=\left(2^{r}-1\right) s^{d} a^{2^{r}}=s^{d} a^{2^{r}}$ since $r \geq 1$. Secondly, we have $S q^{1}\left(t^{2^{r}-1+d}\right)=\left(\begin{array}{c}2^{r}-1+d \\ 1\end{array}\right) t^{2^{r}+d}=t^{2^{r}+d} \neq 0$ since $d$ is even, $r \geq 1$ and $2^{r}+d \leq m$. Thirdly, we have $\varphi\left(s^{d} a^{2^{r}}\right)=0$ by statement (i). From these, we obtain $\varphi\left(s^{d} a^{2^{r}-1}\right) \neq t^{2^{r}-1+d}$, that is, $\varphi\left(s^{d} a^{2^{r}-1}\right)=0$.

Finally we show statement (iii). Let us put $i-2^{r}+1=j$. We note that $2 \leq j \leq 2^{r}-1$. Then we have $s^{d} a^{i}=s^{d} a^{2^{r}-1+j}=S q^{j}\left(s^{d} a^{2^{r}-1}\right)$ since $\left(\begin{array}{c}2^{r}-1 \\ j\end{array}\right)$ is odd. From this, we see that $\varphi\left(s^{d} a^{i}\right)=0$ if $\varphi\left(s^{d} a^{2^{r}-1}\right)=0$.

Now, we are ready to prove Lemma 3.4. By statement (i), we have $\varphi\left(s^{d} a^{i}\right)=$ 0 for $i=1,2$ and also for $i=2^{r}$ with $r \geq 2$. First we assume that $m-d+1$ is not a power of 2 . Then, for any integer $r$ with $r \geq 2$, we have $2^{r}-1+d \neq m$, so that we have $\varphi\left(s^{d} a^{i}\right)=0$ for $i=2^{r}-1$ by (ii). Hence we have $\varphi\left(s^{d} a^{i}\right)=0$ for $i$ with $2^{r}+1 \leq i \leq 2^{r+1}-2$ by (iii). Thus we obtain $\varphi\left(s^{d} a^{i}\right)=0$ for $i$ with $2^{r}-1 \leq i \leq 2^{r+1}-2$ for all $r \geq 2$, and we conclude that $\varphi=0$. This proves part (1) of the lemma.

Next we prove part (2). Assume that $m-d+1$ is a power of 2 , say, $2^{\ell}$. Then we have $2^{\ell}-1+d=m$. Using (i), (ii) and (iii) just as in the previous argument, we obtain $\varphi\left(s^{d} a^{i}\right)=0$ unless $i=2^{\ell}-1$ or $2^{\ell}+1 \leq i \leq 2^{\ell+1}-2$. For dimensional reasons, we also have $\varphi\left(s^{d} a^{i}\right)=0$ in $\widetilde{H}^{*}\left(\mathbb{R} P^{m}\right)$ for $2^{\ell}+1 \leq i \leq 2^{\ell+1}-2$ since $m<i+d$. Therefore, we obtain $\varphi\left(s^{d} a^{i}\right)=0$ unless $i=2^{\ell}-1$. In the case where $m>n+d$, we have $s^{d} a^{2^{\ell}-1}=0$ in $\widetilde{H}^{*}\left(\Sigma^{d} \mathbb{R} P^{n}\right)$ for dimensional reasons, so that we conclude that $\varphi=0$. In the case where $m \leq n+d$, the formula $\varphi\left(s^{d} a^{2^{\ell}-1}\right)=t^{m}$ actually defines the only non-zero homomorphism from $\widetilde{H}^{*}\left(\Sigma^{d} \mathbb{R} P^{n}\right)$ to $\widetilde{H}^{*}\left(\mathbb{R} P^{m}\right)$. Indeed, there is no integer $i$ with $0<i<2^{\ell}-1$ such that $s^{d} a^{2^{\ell}-1}=S q^{i}\left(s^{d} a^{2^{\ell}-1-i}\right)$ since $\left(2^{\ell}-1-i\right)$ is even. This proves part (2) and the proof of Lemma 3.4 is thus completed.

Now we are ready to prove Proposition 3.2.

Proof of Proposition 3.2. Let $\alpha$ be an $m$-dimensional vector bundle over $\Sigma^{d} \mathbb{R} P^{n}$, where $d=2,4$ or 8 .

First, we consider the case where $m<2 d$. Recall that the smallest integer $i$ so that $w_{i}(\alpha)$ is non-zero must be a power of 2 . Since $\operatorname{dim} \alpha<2 d$ and $\Sigma^{d} \mathbb{R} P^{n}$ is $d$-connected, it follows that $w_{i}(\alpha)=0$ for all $i>0$, that is, $W(\alpha)=1$. Hence, by Proposition 2.1 (and the remark after it), we obtain ind $\alpha=m$ as required.

Next, we consider the case where $m>2 d$. If $m-d+1$ is not a power of 2 , we have $\operatorname{Hom}\left(\widetilde{H}^{*}\left(\Sigma^{d} \mathbb{R} P^{n}\right), \widetilde{H}^{*}\left(\mathbb{R} P^{m}\right)\right)=0$ by Lemma 3.4 , so that we obtain ind $\alpha=m$ as required, again by Proposition 2.1 (and the remark after it). Assume that $m-d+1$ is a power of 2 , say, $2^{\ell}$. Then we claim that $w_{m}(\alpha)=0$. 
To show this, we calculate $S q^{d-1} w_{2^{\ell}}(\alpha)$ using the $\mathrm{Wu}$ formula [11]. We have

$$
\begin{aligned}
S q^{d-1} w_{2^{\ell}} & =\sum_{i=0}^{d-1}\left(\begin{array}{c}
2^{\ell}-d+i \\
i
\end{array}\right) w_{d-1-i} w_{2^{\ell}+i} \\
& =\left(\begin{array}{c}
2^{\ell}-1 \\
d-1
\end{array}\right) w_{2^{\ell}+d-1} \\
& =w_{m}
\end{aligned}
$$

where we have abbreviated $w_{i}(\alpha)$ as $w_{i}$. Note that $w_{j}=0$ for $0<j \leq d-1$ since $\Sigma^{d} \mathbb{R} P^{n}$ is $d$-connected, and also note that $\left(\begin{array}{c}2^{\ell}-1 \\ d-1\end{array}\right)$ is odd since $d-1<2^{\ell}-2$ by the assumption $m>2 d$. On the other hand, $w_{2^{\ell}}$ is either zero or $s^{d} a^{2^{\ell}-d}$, and we have

$$
\begin{aligned}
S q^{d-1}\left(s^{d} a^{2^{\ell}-d}\right) & =\left(\begin{array}{c}
2^{\ell}-d \\
d-1
\end{array}\right) s^{d} a^{2^{\ell}-1} \\
& =0
\end{aligned}
$$

since $\left(\begin{array}{c}2^{\ell}-d \\ d-1\end{array}\right)$ is even for $d=2,4$ or 8 . Therefore, whether $w_{2^{\ell}}$ is zero or not, we conclude that $w_{m}=0$. Now, let $g: \mathbb{R} P^{m} \rightarrow \Sigma^{d} \mathbb{R} P^{n}$ be an arbitrary map. By Lemma 3.4, the homomorphism $g^{*}: \widetilde{H}^{*}\left(\Sigma^{d} \mathbb{R} P^{n}\right) \rightarrow \widetilde{H}^{*}\left(\mathbb{R} P^{m}\right)$ is zero except possibly in dimension $m$. Since $w_{m}(\alpha)=0$ by the previous argument, we see that $g^{*}(W(\alpha))=1$. Therefore, by Proposition 2.1, we obtain ind $\alpha=m$. By this, we have completed the proof of part (1) of Proposition 3.2.

Finally, we consider the case where $m=2 d$. Let $g: \mathbb{R} P^{2 d+1} \rightarrow \Sigma^{d} \mathbb{R} P^{n}$ be an arbitrary map and let us consider the homomorphism $g^{*}: \widetilde{H}^{*}\left(\Sigma^{d} \mathbb{R} P^{n}\right) \rightarrow$ $\widetilde{H}^{*}\left(\mathbb{R} P^{2 d+1}\right)$. When $d=4$ or 8 , we have

$$
\operatorname{Hom}\left(\widetilde{H}^{*}\left(\Sigma^{d} \mathbb{R} P^{n}\right), \widetilde{H}^{*}\left(\mathbb{R} P^{2 d+1}\right)\right)=0
$$

by Lemma 3.4 since $(2 d+1)-d+1$ is not a power of 2 . Hence $g^{*}$ is the zero homomorphism for $d=4,8$. When $d=2, g^{*}$ is zero except possibly in dimension $2 d+1$, by Lemma 3.4. Since $\operatorname{dim} \alpha=2 d$, we obviously have $w_{2 d+1}(\alpha)=0$. Therefore we have $g^{*}(W(\alpha))=1$ for $d=2$. Thus we have $g^{*}(W(\alpha))=1$ for $d=2,4$ or 8 , so that we can conclude that ind $\alpha \leq 2 d+1$ by Proposition 2.1. The first half of (2) of Proposition 3.2 is thus obtained.

Now we assume that $n \geq 2 d$ in addition to $m=2 d$. Since $\Sigma^{d} \mathbb{R} P^{n}$ is $d$ connected and the smallest integer $i$ so that $w_{i}(\alpha) \neq 0$ is a power of 2 , the only Whitney class which is possibly non-zero is $w_{2 d}(\alpha)$. Thus we can write as $W(\alpha)=1+w_{2 d}(\alpha)$. Here we claim that the following is true:

Assertion 3.5. Let $d=2,4$ or 8 , and assume that $n \neq 0,6,7 \bmod 8$ when $d=2$. Then we have either $W(\beta)=1$ or $W(\beta)=1+\sum_{i=1}^{n} s^{d} a^{i d}$ for any vector bundle $\beta$ over $\Sigma^{d} \mathbb{R} P^{n}$. 
Clearly we have $s^{d} a^{d} \neq 0$ and $s^{d} a^{2 d} \neq 0$ since $n \geq 2 d$. Hence, admitting that Assertion 3.5 is true, we obtain $W(\alpha) \equiv 1+w_{2 d}(\alpha)=1$ when $d=4$ or 8 . When $d=2$, we also have $W(\alpha) \equiv 1+w_{4}(\alpha)=1$ at least for $n=4$. For $n>4$, let us consider the inclusion map $i: \Sigma^{2} \mathbb{R} P^{4} \hookrightarrow \Sigma^{2} \mathbb{R} P^{n}$. Since $i^{*}: H^{4}\left(\Sigma^{2} \mathbb{R} P^{n}\right) \rightarrow H^{4}\left(\Sigma^{2} \mathbb{R} P^{4}\right)$ is injective, we have $W(\alpha) \equiv 1+w_{4}(\alpha)=1$ also for $n>4$ from the result for $n=4$. Thus we have $W(\alpha)=1$ for $d=2,4$ or 8 , so that we can conclude that ind $\alpha=\operatorname{dim} \alpha$ by Proposition 2.1 (and the remark after it). Therefore the proof of Proposition 3.2 is complete if we prove Assertion 3.5.

Proof of Assertion 3.5. Since the cup product is trivial in $\widetilde{H}^{*}\left(\Sigma^{d} \mathbb{R} P^{n}\right)$, we have $W(2 \beta)=1$ for any vector bundle $\beta$ over $\Sigma^{d} \mathbb{R} P^{n}$, using the Whitney sum formula. According to the results of Fujii [3], we know that $\widetilde{K O}\left(\Sigma^{2} \mathbb{R} P^{n}\right)$ is isomorphic to $\mathbb{Z}_{2}$ if $n \neq 10,6,7(\bmod 8)$, and also that $\widetilde{K O}\left(\Sigma^{4} \mathbb{R} P^{n}\right)$ and $\widetilde{K O}\left(\Sigma^{8} \mathbb{R} P^{n}\right)$ are cyclic groups of order a power of 2 . Therefore, in these cases, there are at most two types of $W(\beta)$, one of which is $W(\beta)=1$. As is shown in the proof of [10, Proposition 3.1], there exists $\beta$ such that $W(\beta)=$ $1+\sum_{i=1}^{n} s^{d} a^{i d}$. Thus the assertion follows and the proof of Proposition 3.2 is completed.

Now we proceed to prove Proposition 3.3.

Proof of Proposition 3.3. In view of Proposition 2.2 and part (2) of Proposition 3.2 , it suffices to prove the following lemma.

Lemma 3.6. Let $d=2$ or 4 , and assume that $d \leq n \leq 2 d-1$. Then there exist maps $f: \Sigma^{d} \mathbb{R} P^{n} \rightarrow S^{2 d}$ and $g: \mathbb{R} P^{2 d} \rightarrow \Sigma^{d} \mathbb{R} \bar{P}^{n}$ such that

$$
(f \circ g)^{*}: H^{2 d}\left(S^{2 d}\right) \longrightarrow H^{2 d}\left(\mathbb{R} P^{2 d}\right)
$$

is an isomorphism.

Proof. First we take, as $f: \Sigma^{d} \mathbb{R} P^{n} \rightarrow S^{2 d}$, the following composition:

$$
\begin{aligned}
\Sigma^{d} \mathbb{R} P^{n} \hookrightarrow \Sigma^{d} \mathbb{R} P^{2 d-1} \stackrel{j}{\rightarrow} \Sigma^{d} \mathbb{R} P_{d}^{2 d-1}=\Sigma^{d}\left(\mathbb{R} P^{d-1}\right)^{d \xi} & \stackrel{h}{\underset{ }{\rightarrow}} \Sigma^{d}\left(\mathbb{R} P^{d-1}\right)^{d} \\
& \stackrel{q}{\rightarrow} \Sigma^{d}(p t)^{d}=S^{2 d},
\end{aligned}
$$

where $j$ is the map collapsing the $(2 d-1)$-skeleton, and $q$ is the map induced from the constant map $\mathbb{R} P^{d-1} \rightarrow p t$, while $h$ is the map obtained from the fact that $d \xi$ is isomorphic to the $d$-dimensional trivial bundle over $\mathbb{R} P^{d-1}$. Then, it is easy to see that $f^{*}: H^{2 d}\left(S^{2 d}\right) \rightarrow H^{2 d}\left(\Sigma^{d} \mathbb{R} P^{n}\right)$ is an isomorphism.

Next, as $g: \mathbb{R} P^{2 d} \rightarrow \Sigma^{d} \mathbb{R} P^{n}$, we take the following composition:

$$
\begin{aligned}
\mathbb{R} P^{2 d} \stackrel{j^{\prime}}{\rightarrow} \mathbb{R} P_{d+1}^{2 d} & =\left(\mathbb{R} P^{d-1}\right)^{(d+1) \xi} \underset{h^{\prime}}{\stackrel{\approx}{\approx}}\left(\mathbb{R} P^{d-1}\right)^{\xi+d} \\
& =\Sigma^{d}\left(\mathbb{R} P^{d-1}\right)^{\xi} \\
& =\Sigma^{d} \mathbb{R} P^{d} \hookrightarrow \Sigma^{d} \mathbb{R} P^{n}
\end{aligned}
$$


where $j^{\prime}$ is the map collapsing the $d$-skeleton, and $h^{\prime}$ is the map obtained from the fact that $d \xi$ is isomorphic to the $d$-dimensional trivial bundle over $\mathbb{R} P^{d-1}$. Then, it is immediate that $g^{*}: H^{2 d}\left(\Sigma^{d} \mathbb{R} P^{n}\right) \rightarrow H^{2 d}\left(\mathbb{R} P^{2 d}\right)$ is an isomorphism. This completes the proof of Lemma 3.6.

Remark. Since $8 \xi$ is isomorphic to the 8-dimensional trivial bundle over $\mathbb{R} P^{7}$, the above lemma is also true for $d=8$. However, Proposition 2.2 is not true for $d=16$, which is the reason why we cannot apply the same argument as in Proposition 3.3 to the case $d=8$.

\section{4. $k$-fold suspension for the case $k=3,5,6$ or 7}

In this section, we consider $\Sigma^{k} \mathbb{R} P^{n}$ with $k=3,5,6$ or 7 and prove parts (3), (5), (6), and (7) of Theorem 1.3.

First, let us consider the case $k=7$. By Theorem $1.2, \Sigma^{7} \mathbb{R} P^{n}$ is not I-trivial only if $n=1$. Since $\Sigma^{7} \mathbb{R} P^{1}\left(=S^{8}\right)$ is not I-trivial, part (7) of Theorem 1.3 is obvious.

Next, we consider the cases $k=5$ and $k=6$. By Theorem $1.2, \Sigma^{5} \mathbb{R} P^{n}$ is not I-trivial only if $n=3$, and $\Sigma^{6} \mathbb{R} P^{n}$ is not I-trivial only if $n=2,3$. Thus, parts (5) and (6) of Theorem 1.3 follow from the following proposition.

\section{Proposition 4.1.}

(1) $\Sigma^{5} \mathbb{R} P^{3}$ is not I-trivial.

(2) $\Sigma^{6} \mathbb{R} P^{n}$ is not I-trivial for $n=2$ or 3 .

Proof. Since $\mathbb{R} P^{3}$ is S-reducible and since the suspension map $\Sigma^{\infty}:\left[S^{5}, \Sigma^{2} \mathbb{R} P^{3}\right]$ $\rightarrow\left\{S^{3}, \mathbb{R} P^{3}\right\}$ is surjective, we see that $\Sigma^{2} \mathbb{R} P^{3}$ is reducible. Hence $\Sigma^{5} \mathbb{R} P^{3}$ is also reducible, so that there exists a map $g: S^{8} \rightarrow \Sigma^{5} \mathbb{R} P^{3}$ such that the composite

$$
S^{8} \stackrel{g}{\longrightarrow} \Sigma^{5} \mathbb{R} P^{3} \stackrel{f}{\longrightarrow} S^{8}
$$

is homotopic to the identity map. Here $f$ is the map which collapses the 7skeleton. As in the proof of Proposition 2.2, we consider the vector bundle $\alpha=$ $f^{*}(\rho)$, where $\rho$ is the Hopf vector bundle over $S^{8}$. Since $g^{*}(\alpha)=i d^{*}(\rho)=\rho$, we obtain a $\mathbb{Z}_{2}$-map $S^{15}=S(\rho) \rightarrow S(\alpha)$ by restricting the bundle monomorphism $g: \rho \rightarrow \alpha$ to the sphere bundles. Therefore we have ind $\alpha \geq 16$. Since $\operatorname{dim} \alpha=8$, it follows that $\Sigma^{5} \mathbb{R} P^{3}$ is not I-trivial and part (1) of the proposition is obtained.

Next let $n=2$ or 3 , and consider $\Sigma^{6} \mathbb{R} P^{n}$. We use Proposition 2.2. As a map $f: \Sigma^{6} \mathbb{R} P^{n} \rightarrow S^{8}$, when $n=2$, we take the quotient map which collapses the 7-skeleton. When $n=3$, we take the composite map

$$
\Sigma^{6} \mathbb{R} P^{3} \rightarrow S^{8} \cup e^{9} \simeq S^{8} \vee S^{9} \rightarrow S^{8},
$$

where the first map collapses the 7 -skeleton and the last map is the projection. As a map $g: \mathbb{R} P^{8} \rightarrow \Sigma^{6} \mathbb{R} P^{n}$, when $n=2$, we take the composite map

$$
\mathbb{R} P^{8} \rightarrow S^{7} \cup_{2} e^{8} \approx \Sigma^{6} \mathbb{R} P^{2},
$$


where the first map collapses the 6 -skeleton. When $n=3$, we take the composite of this map with the inclusion $i: \Sigma^{6} \mathbb{R} P^{2} \hookrightarrow \Sigma^{6} \mathbb{R} P^{3}$. Taking $f$ and $g$ as above, $f^{*}: H^{8}\left(S^{8}\right) \rightarrow H^{8}\left(\Sigma^{6} \mathbb{R} P^{n}\right)$ and $g^{*}: H^{8}\left(\Sigma^{6} \mathbb{R} P^{n}\right) \rightarrow H^{8}\left(\mathbb{R} P^{8}\right)$ are both isomorphisms. Therefore, part (2) of the proposition is obtained from Proposition 2.2.

Finally, in this section, we consider $\Sigma^{3} \mathbb{R} P^{n}$, the case $k=3$. By Theorem 1.2, $\Sigma^{3} \mathbb{R} P^{n}$ is not I-trivial only if $n=1,5$. Clearly $\Sigma^{3} \mathbb{R} P^{1}\left(=S^{4}\right)$ is not I-trivial, and thus part (3) of Theorem 1.3 is obvious. It is still open whether $\Sigma^{3} \mathbb{R} P^{5}$ is I-trivial or not. However, we have a partial result as follows.

\section{Proposition 4.2.}

(1) Let $\alpha$ be a vector bundle over $\Sigma^{3} \mathbb{R} P^{5}$ with $\operatorname{dim} \alpha=m$.

(a) If $m \neq 8,9$, then we have ind $\alpha=m$.

(b) If $m=8$ or 9 , then we have $m \leq$ ind $\alpha \leq 10$.

(2) $\Sigma^{3} \mathbb{R} P^{5}$ is not I-trivial if and only if there exists a map $g: \mathbb{R} P^{8} \rightarrow$ $\Sigma^{3} \mathbb{R} P^{5}$ such that $g^{*}: H^{8}\left(\Sigma^{3} \mathbb{R} P^{5}\right) \rightarrow H^{8}\left(\mathbb{R} P^{8}\right)$ is an isomorphism.

Proof. By Proposition 2.1 (and the remark after it), part (1) of the proposition immediately follows from the following two assertions:

(i) If $m<8$, we have $W(\alpha)=1$.

(ii) If $m \geq 10$, we have $\operatorname{Hom}\left(\widetilde{H}^{*}\left(\Sigma^{3} \mathbb{R} P^{5}\right), \widetilde{H}^{*}\left(\mathbb{R} P^{m}\right)\right)=0$.

We show that these assertions are true. Since $\Sigma^{3} \mathbb{R} P^{5}$ is 3 -connected, we have $w_{i}(\alpha)=0$ for $0<i<4$. Hence, by Lemma 3.3 of [10], we have $S q^{1} w_{4}(\alpha)=0$. On the other hand, we have $S q^{1}\left(s^{3} a\right) \neq 0$. Therefore we obtain $w_{4}(\alpha) \neq s^{3} a$, that is, $w_{4}(\alpha)=0$. Since the smallest integer $k$ so that $w_{k}(\alpha) \neq 0$ is a power of 2 , it follows that $w_{i}(\alpha)=0$ for $i<8$ and assertion (i) follows. Assume that $m \geq 10$ and let $\varphi: \widetilde{H}^{*}\left(\Sigma^{3} \mathbb{R} P^{5}\right) \rightarrow \widetilde{H}^{*}\left(\mathbb{R} P^{m}\right)$ be an arbitrary homomorphism. Since $S q^{4}\left(s^{3} a\right)=0$ and $S q^{4} t^{4}=t^{8} \neq 0$, we obtain $\varphi\left(s^{3} a\right) \neq t^{4}$, that is, $\varphi\left(s^{3} a\right)=0$. Similarly, since $S q^{4}\left(s^{3} a^{3}\right)=0$ and $S q^{4} t^{6}=t^{10} \neq 0$, we obtain $\varphi\left(s^{3} a^{3}\right)=0$. Since $s^{3} a^{2}=S q^{1}\left(s^{3} a\right), s^{3} a^{4}=S q^{1}\left(s^{3} a^{3}\right)$ and $s^{3} a^{5}=S q^{2}\left(s^{3} a^{3}\right)$, we see that $\varphi\left(s^{3} a^{j}\right)=0$ for all $j(1 \leq j \leq 5)$, so that assertion (ii) follows.

Next we prove part (2) of the proposition. The "if" part is immediate from Proposition 2.2, taking as $f: \Sigma^{3} \mathbb{R} P^{5} \rightarrow S^{8}$ the map which collapses the 7-skeleton. Suppose that $\Sigma^{3} \mathbb{R} P^{5}$ is not I-trivial, and let $\alpha$ be a vector bundle over $\Sigma^{3} \mathbb{R} P^{5}$ such that ind $\alpha>\operatorname{dim} \alpha$. We put $m=\operatorname{dim} \alpha$. Then $m$ must be either 8 or 9 by part (1). From Proposition 2.1, there exists a map $g: \mathbb{R} P^{m} \rightarrow \Sigma^{3} \mathbb{R} P^{5}$ such that $g^{*}(W(\alpha)) \neq 1$. Here, we recall that we have $w_{i}(\alpha)=0$ for $i<8$. Also, we have $w_{9}(\alpha)=0$ for dimensional reasons. Thus, we see that $g^{*}\left(w_{8}(\alpha)\right)=t^{8}$. Therefore, when $m=8, g$ is the desired map. When $m=9$, the composite of $g$ and the inclusion $\mathbb{R} P^{8} \hookrightarrow \mathbb{R} P^{9}$ gives the desired map. This proves the "only if" part, and the proof is complete.

Remark. It is easy to see that

$$
\operatorname{Hom}\left(\widetilde{H}^{*}\left(\Sigma^{3} \mathbb{R} P^{5}\right), \widetilde{H}^{*}\left(\mathbb{R} P^{8}\right)\right) \cong \mathbb{Z}_{2}
$$


and its only non-zero homomorphism $\varphi$ is given by

$$
\varphi\left(s^{3} a^{j}\right)= \begin{cases}t^{j+3} & (j=3,5) \\ 0 & (j \neq 3,5) .\end{cases}
$$

Proposition 4.2 implies that this homomorphism is realizable by a map if and only if $\Sigma^{3} \mathbb{R} P^{5}$ is not I-trivial.

\section{5. $k$-fold suspension for $k=1$}

In this section, we consider $\Sigma \mathbb{R} P^{n}$ and prove part (1) of Theorem 1.3, that is, the following proposition.

Proposition 5.1. $\Sigma \mathbb{R} P^{n}$ is not I-trivial for $n=1,2$ or 3 .

Proof. The proposition is clearly true when $n=1$ since $\Sigma \mathbb{R} P^{1}=S^{2}$. By Theorem 1.2 of [8], it is also true when $n=2$. Furthermore, there is a 3 dimensional bundle $\alpha_{3}$ over $\Sigma \mathbb{R} P^{2}$ such that ind $\alpha_{3}=4$ (see [8, Theorem 4.1]). Let

$$
S^{3} \stackrel{p}{\longrightarrow} \Sigma \mathbb{R} P^{2} \stackrel{i}{\longrightarrow} \Sigma \mathbb{R} P^{3}
$$

be the standard cofibration and let us consider the exact sequence

$$
\left[S^{3}, B O(3)\right] \stackrel{p^{*}}{\longleftarrow}\left[\Sigma \mathbb{R} P^{2}, B O(3)\right] \stackrel{i^{*}}{\longleftarrow}\left[\Sigma \mathbb{R} P^{3}, B O(3)\right] .
$$

Since $\left[S^{3}, B O(3)\right] \cong \pi_{2}(O(3))=0, i^{*}$ is surjective. Hence, we can take $\beta \in$ $\left[\Sigma \mathbb{R} P^{3}, B O(3)\right]$ so that $i^{*} \beta=\alpha_{3}$. Then we have ind $\beta \geq$ ind $i^{*} \beta=4$, which implies that $\Sigma \mathbb{R} P^{3}$ is not I-trivial.

It is still open for $n \geq 4$ whether $\Sigma \mathbb{R} P^{n}$ is I-trivial or not. For a positive integer $m$, let $\lambda(m)$ denote the largest integer $r$ such that $2^{r} \leq m$. We have the following proposition.

Proposition 5.2. Let $n, m$ be positive integers and let $\alpha$ be a vector bundle over $\Sigma \mathbb{R} P^{n}$ with $\operatorname{dim} \alpha=m$. Then we have ind $\alpha=m$ unless $n \leq m<2^{\lambda(n+1)+1}$. When $n \neq 3,7$, we also have ind $\alpha=m$ for $n=m$.

The following lemma, which is an analogue of Lemma 3.4, shows that ind $\alpha=$ $m$ if $m \geq 2^{\lambda(n+1)+1}$.

Lemma 5.3. For positive integers $n$ and $m$, we have

$$
\operatorname{Hom}\left(\widetilde{H}^{*}\left(\Sigma \mathbb{R} P^{n}\right), \widetilde{H}^{*}\left(\mathbb{R} P^{m}\right)\right) \cong \begin{cases}0 & \left(m \geq 2^{\lambda(n+1)+1}\right), \\ \mathbb{Z}_{2} & \left(m<2^{\lambda(n+1)+1}\right) .\end{cases}
$$

In the latter case, the non-zero homomorphism is non-zero only in dimension $2^{\lambda(m)}$

Proof. Let $\varphi: \widetilde{H}^{*}\left(\Sigma \mathbb{R} P^{n}\right) \rightarrow \widetilde{H}^{*}\left(\mathbb{R} P^{m}\right)$ be a homomorphism. We put $\lambda(n+$ $1)=r$ and $\lambda(m)=\ell$, that is, $2^{r} \leq n+1<2^{r+1}$ and $2^{\ell} \leq m<2^{\ell+1}$. The lemma follows from the following two assertions:

(i) If $i+1$ is not a power of 2 , then $\varphi\left(s a^{i}\right)=0$. 
(ii) If $i+1=2^{j}$ and $j \neq \ell$, then $\varphi\left(s a^{i}\right)=0$.

In fact, from (i) and (ii) we have $\varphi\left(s a^{i}\right)=0$ unless $i=2^{\ell}-1$. In the case where $m \geq 2^{r+1}$, we have $n+1<2^{r+1} \leq 2^{\ell}$, so that we have $s a^{2^{\ell}-1}=0$ in $\widetilde{H}^{*}\left(\Sigma \mathbb{R} P^{n}\right)$ for dimensional reasons. Therefore we obtain $\varphi=0$ in this case. In the case where $m<2^{r+1}$, we have $2^{\ell} \leq 2^{r} \leq n+1$, and the formula $\varphi\left(s a^{2^{\ell}-1}\right)=t^{2^{\ell}}$ actually defines a homomorphism from $\widetilde{H}^{*}\left(\Sigma \mathbb{R} P^{n}\right)$ to $\widetilde{H}^{*}\left(\mathbb{R} P^{m}\right)$, since there is no integer $i$ with $0<i<2^{\ell}-1$ such that $s a^{2^{\ell}-1}=S q^{i}\left(s a^{2^{\ell}-1-i}\right)$. Thus the lemma follows from assertions (i) and (ii).

Now we prove assertions (i) and (ii). Let us write $i+1=2^{j}+k$ with $0<k<2^{j}$. Then we have $\varphi\left(s a^{i}\right)=\varphi\left(S q^{k}\left(s a^{2^{j}-1}\right)\right)=S q^{k}\left(\varphi\left(s a^{2^{j}-1}\right)\right)$, since $\left(\begin{array}{c}2^{j}-1 \\ k\end{array}\right)$ is odd. Here, if $\varphi\left(s a^{2^{j}-1}\right)=0$, we clearly have $\varphi\left(s a^{i}\right)=0$, while if $\varphi\left(s a^{2^{j}-1}\right)=t^{2^{j}}$, we have $\varphi\left(s a^{i}\right)=S q^{k}\left(t^{2^{j}}\right)=0$, since $\left(\begin{array}{c}2^{j} \\ k\end{array}\right)$ is even. Therefore, whether $\varphi\left(s a^{2^{j}-1}\right)$ is zero or not, we obtain $\varphi\left(s a^{i}\right)=0$. Thus assertion (i) follows.

Next, let $i+1=2^{j}$ and $j \neq \ell$. In the case where $j \geq \ell+1$, we have $\varphi\left(s a^{2^{j}-1}\right)=0$ in $\widetilde{H}^{*}\left(\mathbb{R} P^{m}\right)$ for dimensional reasons. In the case where $j \leq \ell-1$, we have $S q^{2^{j}}\left(t^{2^{j}}\right)=t^{2^{j+1}} \neq 0$, since $j+1 \leq \ell$. On the other hand, we obviously have $S q^{2^{j}}\left(s a^{2^{j}-1}\right)=0$. From these, we obtain $\varphi\left(s a^{2^{j}-1}\right) \neq t^{2^{j}}$, that is, $\varphi\left(s a^{2^{j}-1}\right)=0$ also in this case. Thus assertion (ii) follows and the proof of the lemma is complete.

Proof of Proposition 5.2. When $m \geq 2^{\lambda(n+1)+1}$, we have ind $\alpha=m$ by the above lemma, using Proposition 2.1 (and the remark after it). To show that ind $\alpha=m$ when $m<n$, we investigate possibilities of types of Stiefel-Whitney classes just like Assertion 3.5. By [3, Theorem 1], we have

$$
\widetilde{K O}\left(\Sigma \mathbb{R} P^{n}\right) \cong \begin{cases}\mathbb{Z}+\mathbb{Z}_{2} & \text { if } n \equiv 3 \bmod 4 \\ \mathbb{Z}_{2} & \text { if } n \neq 3 \bmod 4 .\end{cases}
$$

Moreover, when $n \equiv 3(\bmod 4)$, we have the following exact sequence:

$$
0 \longleftarrow \widetilde{K O}\left(\Sigma \mathbb{R} P^{n-1}\right) \stackrel{i^{*}}{\longleftarrow} \widetilde{K O}\left(\Sigma \mathbb{R} P^{n}\right) \stackrel{j^{*}}{\longleftarrow} \widetilde{K O}\left(S^{n+1}\right)=\mathbb{Z} \longleftarrow 0 .
$$

Recall that $S^{n+1}$ is W-trivial if $n \neq 1,3,7$ (see [5]). Since the cup product is trivial in $\widetilde{H}^{*}\left(\Sigma \mathbb{R} P^{n}\right)$, we see that there are at most three types of non-trivial Stiefel-Whitney classes if $n=3$ or 7 , while there is at most one type if $n \neq 3,7$. On the other hand, by [10, Proposition 3.1], there is a vector bundle over $\Sigma \mathbb{R} P^{n}$ whose Stiefel-Whitney class is $1+\sum_{i=1}^{n} s a^{i}$. It follows that the possibilities are $1+s a^{n}, 1+\sum_{i=1}^{n} s a^{i}$ and $1+\sum_{i=1}^{n-1} s a^{i}$ when $n=3$ or 7 , while the only possibility is $1+\sum_{i=1}^{n} s a^{i}$ when $n \neq 3,7$.

Now, we show that ind $\alpha=m$ when $m<n$. Since $m<n$, we have $w_{i}(\alpha)=0$ for $i=n$ and $i=n+1$, for dimensional reasons. From the above possibilities of Stiefel-Whitney classes, we must have $W(\alpha)=1$, so that we obtain ind $\alpha=m$. 
Likewise, we obtain ind $\alpha=m$ when $m=n$ and $n \neq 3,7$. This completes the proof.

\section{References}

[1] P. E. Conner and E. E. Floyd, Fixed point free involutions and equivariant maps, Bull. Amer. Math. Soc. 66 (1960), 416-441.

[2] _ Fixed point free involutions and equivariant maps II, Trans. Amer. Math. Soc. 105 (1962), 222-228.

[3] M. Fujii, $K_{O}$-groups of projective spaces, Osaka J. Math. 4 (1967), 141-149.

[4] F. Hirzebruch, Neue Topologische Methoden in der Algebraischen Geometrie, Ergebnisse der Math., Springer, 1956.

[5] J. Milnor, Some consequences of a theorem of Bott, Ann. of Math. (2) 68 (1958), 444449.

[6] R. Tanaka, On the index and co-index of sphere bundles, Kyushu J. Math. 57 (2003), no. $2,371-382$.

[7] _ A stability theorem for the index of sphere bundles, Bull. Belg. Math. Soc. Simon Stevin 14 (2007), no. 1, 177-182.

[8] _ A fiberwise analogue of the Borsuk-Ulam theorem for sphere bundles over a 2-cell complex, Topology Appl. 154 (2007), no. 8, 1827-1833.

[9] _ A Borsuk-Ulam type theorem for sphere bundles over stunted projective spaces, Topology Appl. 156 (2009), no. 5, 932-938.

[10] _ On trivialities of Stiefel-Whitney classes of vector bundles over iterated suspension spaces, Homology, Homotopy Appl. 12 (2010), no. 1, 357-366.

[11] W.-T. Wu, Les i-carrés dans une variété grassmannienne, C. R. Acad. Sci. Paris 230 (1950), 918-920.

Department of Mathematics

FACUlty of SCIEnCe And TeChNology

TOKyo University of SCIENCE

NODA, CHIBA, 278-8510, JAPAN

E-mail address: tanaka_ryuichi@ma.noda.tus.ac.jp 